\title{
Effects of microRNA-21 on the interleukin 12/ signal transducer and activator of transcription 4 signaling pathway in asthmatic mice
}

\author{
SONG-QUAN WU ${ }^{l}$, GUANG-LI WANG ${ }^{l}$, LING-YUN LI $I^{2}$ JIAN JI \\ ${ }^{1}$ Medical College of Lishui University, Lishui, PR China \\ ${ }^{2}$ The People's Hospital of Lishui City, PR China \\ ${ }^{3}$ Zhejiang University School of Medicine, Hangzhou, RP China
}

\begin{abstract}
Objective: To study the effect of microRNA-21 (miRNA-21) on the regulation of the interleukin 12 (IL-12)/signal transducer and activator of transcription 4 (STAT4) pathway in the lung tissue of asthmatic mice.

Material and methods: Forty five male C57BL/6 mice were randomly divided into three groups of 15 mice each: normal control, asthmatic model, and dexamethasone. Our mouse model of allergic asathma was established using OVA sensitization and challenge. Hematoxylin and eosin staining was performed to observe the pathological changes in lung tissue morphology. Both the total cell number and the amount of eosinophils (EOS) in the bronchoalveolar lavage fluid (BALF) were manually counted. The expression of miRNA-21 was detected by real time quantitative PCR. The expression levels of IL-12 and STAT4 in lung tissue were assayed via western blot, and immunohistochemistry was used to observe the distribution of their expression.

Results: The expression levels of miRNA-21 as well as the total number of BALF cells and EOS were significantly higher in the asthmatic model group than in the control or dexamethasone groups, with significantly higher amounts found in the dexamethasone group than in the control group. The expression levels of IL-12 and STAT4 proteins were lower in the asthmatic model group than in the control and dexamethasone groups, with a significantly lower expression of IL-12 and STAT4 in the dexamethasone group than in the control group.

Conclusions: The expression level of miRNA-21 was significantly increased and the expression level of IL-12 and STAT4 proteins was significantly decreased in allergic asthmatic mice compared with normal control mice. These findings suggest a role for miRNA-21 and the IL-12/STAT4 pathway in the development of allergic asthma.
\end{abstract}

Key words: allergic asthma, miRNA-21, IL-12, signal transducer and activator of transcription 4 (STAT4).

(Centr Eur J Immunol 2014; 39 (1): 40-45)

\section{Introduction}

Bronchial asthma is a common disease of the respiratory system [1]. The main clinical symptoms of this disease include episodic wheezing, shortness of breath, chest tightness, and coughing. A small number of patients complain of chest pain and present with other clinical manifestations following contact with irritating substances or allergens [2, 3]. It has been shown that the interleukin 12 (IL-12)/signal transducer and transcription activator 4 (STAT4) signal transduction pathway is involved in the development of asthma [4-6]. Expression of IL-12 can be regulated by microRNA-21 (miRNA-21) in IL-4 transgenic mice [7-9]; however, it is not known whether miRNA-21 influences the IL-12/STAT4 signaling pathway in asthmatic mice.

In this paper, we used an established asthmatic mouse model to investigate the effects of miRNA-21 on the regulation of the IL-12/STAT4 pathway during allergic asthma. We observed pathological changes in lung tissue morphology, counted the total cells and EOS cells in BALF, and detected the expression of miRNA-21. We also investigated the expression levels of IL-12 and STAT4 in lung tissue of an asthmatic mouse model. Our aims were to study the effect of miRNA-21 regulation on the IL-12/STAT4 pathway in lung tissue of asthmatic mice and the possible effects of miRNA-21 expression levels on the occurrence and development of asthma. 


\section{Material and methods}

\section{Mice}

We purchased 45 specific pathogen-free (SPF) male mice with a C57BL/6 background (20-22 g) from Shanghai SLAC Laboratory Animal Co., Ltd. (China). The mice used in the studies were sacrificed when they were 6-8-weeks-old.

\section{Laboratory equipment}

Real-time polymerase chain reaction (qPCR) thermal cycler (Agilent-Stratagene, USA); electrophoresis apparatus (BioRad, USA); western blot transfer unit (BioRad, USA); electric atomizer (GSK, UK); microplate reader (AI, USA); optical microscope (Olympus, Japan).

\section{Establishment of asthmatic mouse model}

Mice were housed under SPF conditions at $24 \pm 2^{\circ} \mathrm{C}$ and $55 \pm 5 \%$ relative humidity with a 12 -h light-dark cycle. A commercial diet and potable water were available ad libitum. All experiments were approved by the Animal Research Ethics Board of Lishui University (Lishui, Zhejiang Province, China). Mice were randomly divided into three groups ( $n=15$ mice per group): the model group; the dexamethasone treatment group; and the control group. On days 0 and 8 , mice were administered ovalbumin (OVA; Sigma, USA) dissolved in $1.0 \mathrm{ml}$ of aluminum hydroxide gel (60 $\mu \mathrm{g}$ of OVA and $2.25 \mathrm{mg}$ of aluminum hydroxide gel) by intraperitoneal (i.p.) injection. Commencing on day 15 , mice in the model group received an ultrasonic nebulization of $2 \%$ OVA in a $5 \mathrm{ml}$ solution for $30 \mathrm{~min}$ every other day over 7 days. Mice in the dexamethasone treatment group received an i.p. injection of $0.3 \mathrm{mg} / \mathrm{kg}$ dexamethasone (Sigma, USA) prior to OVA nebulization. Mice in the control group received saline instead of OVA.

\section{Hematoxylin/eosin (HE) staining of lung tissue}

A portion of the right upper lung tissue was obtained, fixed with $4 \%$ paraformaldehyde, dehydrated and embedded in paraffin, then sectioned (5- $\mu \mathrm{m}$ thickness). Sections were baked for $1 \mathrm{~h}$ at $60^{\circ} \mathrm{C}$, hydrated through a graded ethanol series, and stained for $10 \mathrm{~min}$ with hematoxylin, then stained for $3 \mathrm{~min}$ with eosin. After washing under running tap water, sections were dehydrated through a graded alcohol series, treated with xylene, and mounted with neutral gum. Pathological changes in sections were observed using an optical microscope.

\section{Cell count within bronchoalveolar lavage fluid (BALF)}

Mice in all groups were anaesthetized through an i.p. injection of $5 \mathrm{ml} / \mathrm{kg} 20 \%$ urethane solution after the last OVA nebulization event. Serum and left lung tissue was collected from each mouse; bronchoalveolar lavage was conducted and BALF collected. The total cell number and number of eosinophils (EOS) were counted using a microscope and a high power field-of-view.

\section{Real-time polymerase chain reaction analysis of microRNA-21 expression in lung tissue}

A portion of the right lung from each mouse was obtained, and total RNA extracted using Trizol (Invitrogen, USA). We used electrophoresis to determine the quality of the extracted RNA by observing bands corresponding to $28 \mathrm{~S}, 18 \mathrm{~S}$ and $5 \mathrm{~S}$ RNA. The purity and quantity of RNA samples was determined by measuring the absorbance at 260 (A260) and $280 \mathrm{~nm}$ (A280) and calculating the A260/280 ratio. Only samples with an A260/280 ratio greater than 1.8 were used. We used $1 \mu \mathrm{g}$ of total RNA for reverse transcription reactions. The generated cDNA $(1 \mathrm{ml})$ was used as a template in qPCR assays which comprised $25 \mathrm{ml}$. A melt curve for each amplicon was created to determine the specificity of the amplification reaction. We used U6 SnRNA as an internal control.

\section{Western blotting analysis of interleukin 12 and signal transducer and activator of transcription 4 in lung tissue}

Proteins were extracted from the lower right section of lung tissues following homogenization of the tissue and the application of a lysis buffer. The concentration of proteins in each sample was determined, and then samples were subjected to polyacrylamide gel electrophoresis (PAGE) for $2 \mathrm{~h}$ at $100 \mathrm{~V}$. Proteins were transferred to membranes $(0.5 \mathrm{~A}, 60 \mathrm{~min})$ and probed with goat anti-mouse IL-12 ( $1: 100$ dilution; Santa Cruz Biotechnology, USA) and goat anti-mouse STAT4 (1 : 100; Santa Cruz Biotechnology, USA) antibodies at $4{ }^{\circ} \mathrm{C}$ overnight. Samples were repeatedly washed with phosphate-buffered saline (PBS) for 5 min, then incubated with a horseradish peroxidase ( $\mathrm{HrP})$-conjugated rabbit anti-goat antibody (1:4000; Sigma, USA) at room temperature for $1 \mathrm{~h}$. Positive signals were detected using chemiluminescence techniques. We used $\beta$-tubulin as an internal control.

\section{Immunohistochemical analysis of interleukin 12 and signal transducer and activator of transcription 4 in lung tissues}

Formalin-fixed paraffin-embedded lung tissue sections were de-waxed, then rehydrated through a graded alcohol series. Sections were treated with $3 \%(\mathrm{v} / \mathrm{v}) \mathrm{H}_{2} \mathrm{O}_{2}$ to inhibit endogenous peroxidase activity. The antigen was retrieved by microwaving, and sections blocked using normal sheep serum. Sections were then probed with IL-12- and STAT4-specific primary (1 : 100; Santa Cruz Biotechnology, USA) and secondary (1 : 4000; Sigma, USA) antibodies. We added SP (Jiangsu Beyotime, China) reagent and 3,3'-diaminobenzidine (DAB; Beyotime, Jiangsu, China) 


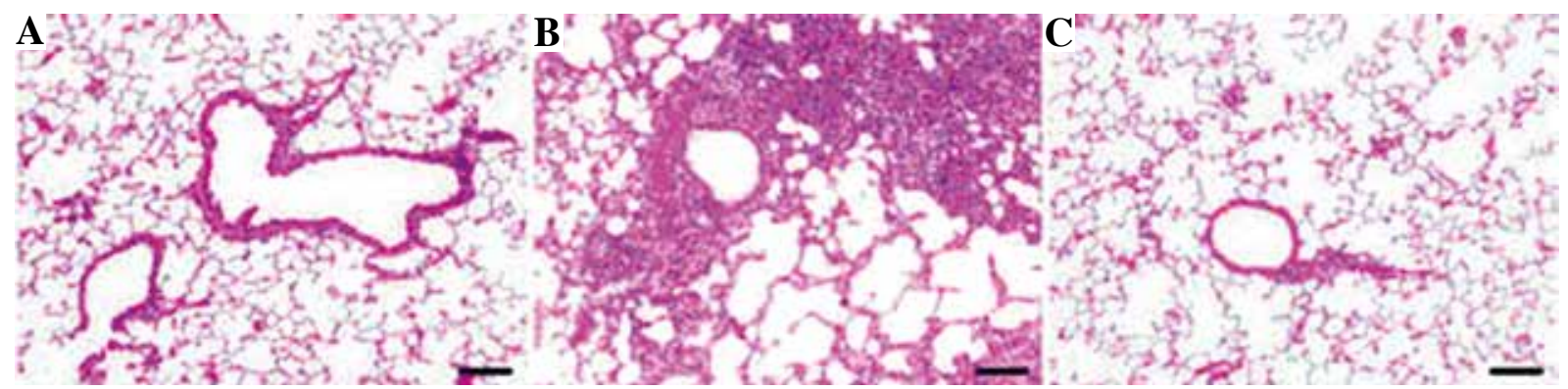

Fig. 1. Lung tissue pathology in the model, dexamethasone, and control groups as determined by HE staining $(\times 200$ magnification). A) control group; B) model group; and C) dexamethasone group. The scale bar indicates $50 \mathrm{~mm}$

to visualize positive brown signals. Nuclear staining was conducted using hematoxylin. Sections were dehydrated and mounted with gum, then examined using a microscope. Differences in protein expression levels among different groups were compared by calculating the average gray value.

\section{Statistical analysis}

Data were analyzed using SPSS18.8 (SPSS Inc., USA). The values we have presented are the mean \pm standard deviation $(X \pm s)$. Single factor analysis of variance (ANOVA) was used for data comparison among groups. A Bonferroni test was used to determine homogeneity of variance, and Dunnett's T3 analysis was used when variance was missing. A $P$-value of less than 0.05 was considered statistically significant.

\section{Results}

\section{Pathological changes in lung tissues}

Alveolar wall tissues of mice in the control group had an integral structure. The alveolar cavity exhibited no exudation, and the alveolar wall had not thickened (Fig. 1A). The alveolar wall structure in the model group was damaged, with the alveolar wall thickened, and a large number of inflammatory cells had infiltrated around the bronchus

Table 1. Comparison of bronchoalveolar lavage fluid (BALF) cell counts among the model, dexamethasone, and control groups

\begin{tabular}{lcc}
\hline Group & $\begin{array}{c}\text { Total cells in } \\
\text { BALF }\left(\times \mathbf{1 0}^{4}\right)\end{array}$ & $\begin{array}{c}\text { No. of EOS in } \\
\text { BALF } \\
(\times \mathbf{4 0 0} \text { photo })\end{array}$ \\
\hline model group & $86.39 \pm 22.53^{*}$ & $241.78 \pm 49.23^{*}$ \\
\hline dexamethasone group & $28.13 \pm 7.39^{* \#}$ & $78.63 \pm 22.51^{* \#}$ \\
\hline control group & $9.46 \pm 3.07$ & $23.56 \pm 5.97$ \\
\hline$F$ & 126.75 & 195.40 \\
\hline$P$ & $<0.05$ & $<0.05$ \\
\hline $\begin{array}{l}\text { *compared with the control group, } p<0.05 ; \text { \#compared with the model } \\
\text { group, } p<0.05\end{array}$ &
\end{tabular}

(Fig. 1B). Lung tissue alveolar wall damage and inflammatory cell infiltration in the dexamethasone group were decreased compared with that in the model group (Fig. 1C).

\section{Bronchoalveolar lavage fluid results}

The number of cells in BALF samples from the control, model, and dexamethasone groups is presented in Table 1. The total cell and EOS numbers in BALF samples in the model group were significantly higher than those in mice of the control group. These values were significantly reduced $(p<0.05)$ following dexamethasone intervention, but still higher than those in normal mice.

\section{Expression profile of microRNA-21 in lung tissues}

Expression levels of miRNA-21 mRNA in the control, model, and dexamethasone groups are presented in Table 2. The miRNA-21 expression levels in asthmatic mice from the model group were significantly higher $(p<0.05)$ than those in normal mice from the control group. Expression levels of miRNA-21 were significantly lower $(p<0.05)$ in mice treated with dexamethasone, but still higher than those in normal mice.

\section{Expression of interleukin 12 and signal transducer and activator of transcription 4 in lung tissues}

Lung IL-12 and STAT4 protein expression levels are presented in Fig. 2, and integral optical density ratios compared

Table 2. Expression levels of miRNA-21 in mice from the model, dexamethasone, and control groups

\begin{tabular}{lc}
\hline Item & MicroRNA-21 levels \\
\hline model group & $4.95 \pm 1.21^{*}$ \\
\hline dexamethasone group & $2.21 \pm 0.67 * \#$ \\
\hline control group & $1.02 \pm 0.23$ \\
\hline$F$ & 92.967 \\
\hline$P$ & $<0.05$ \\
\hline compared with the control group, $p<0.05 ;{ }^{*}$ compared with the model & $<0.05$
\end{tabular}




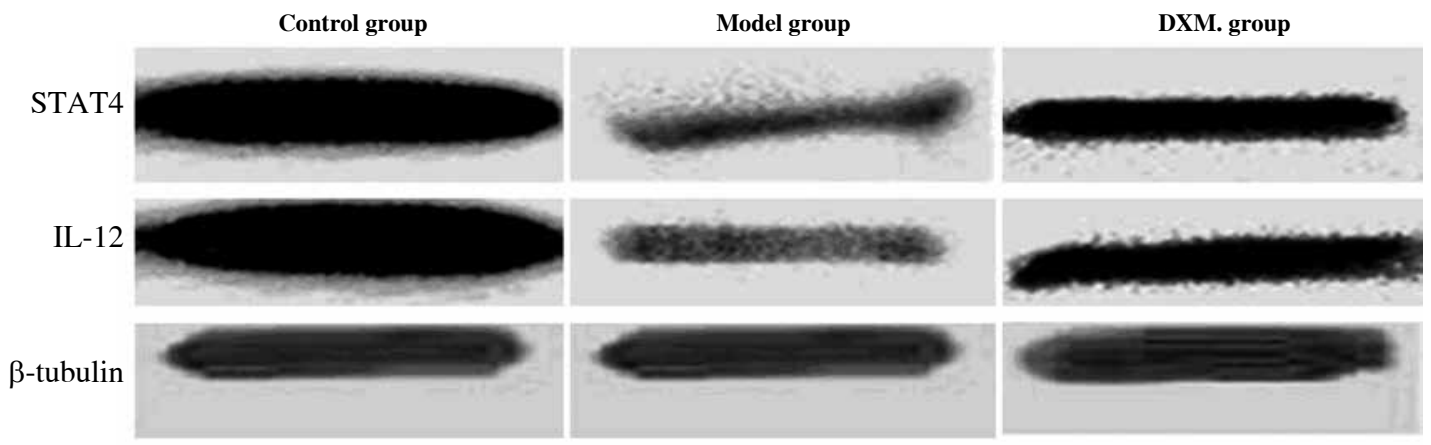

Fig. 2. Detection of interleukin 12 (IL-12) and signal transducer and activator of transcription 4 (STAT4) using western blotting

in Table 3. Interleukin 12 and STAT4 levels in asthmatic mice of the model group were significantly reduced $(p<0.05)$ compared with control mice. Interleukin 12 and STAT4 protein expression levels were significantly improved $(p<0.05)$ following glucocorticoid intervention; however, these levels were still lower than those in control mice.

\section{Immunohistochemical detection of interleukin 12 and signal transducer and activator of transcription 4 in lung tissues}

The expression of IL-12 and STAT4 in the lung tissues of asthmatic mice from the model group were significantly decreased $(p<0.05)$ compared with those in the control group. Expression levels were significantly enhanced $(p<0.05)$ following dexamethasone intervention. Interleukin 12 was expressed in the cytoplasm, and STAT4 was expressed mainly in the nucleus with some expression seen in the cytoplasm (Figs. 3 and 4).

\section{Discussion}

Research has shown that a variety of miRNAs can affect the occurrence and development of asthma [10, 11]. Wang
Table 3. Relative integral optical density values for interleukin 12 (IL-12) and signal transducer and activator of transcription 4 (STAT4) in mice from the model, dexamethasone, and control groups

\begin{tabular}{lcc}
\hline Item & IL-12 & STAT4 \\
\hline model group & $1.32 \pm 0.25^{*}$ & $1.69 \pm 0.31^{*}$ \\
\hline dexamethasone group & $2.98 \pm 0.78^{*} \#$ & $3.48 \pm 0.87^{*} \#$ \\
\hline control group & $4.26 \pm 0.97$ & $5.34 \pm 1.02$ \\
\hline $\mathrm{F}$ & 60.67 & 79.17 \\
\hline $\mathrm{P}$ & $<0.05$ & $<0.05$ \\
\hline *compared with the control group,$p<0.05$ * $^{*}$ compared with the model
\end{tabular}
group, $p<0.05$.

et al. [12] showed that miRNAs can lead to degradation of mRNA and inhibit protein translation. These miRNAs bind to corresponding target sites on the 3'-untranslated region (UTR) of the mRNA, thereby achieving post-translational regulation of gene transcription. Changes in the expression levels of miRNA133a [13], miRNA26a [14] and miR-

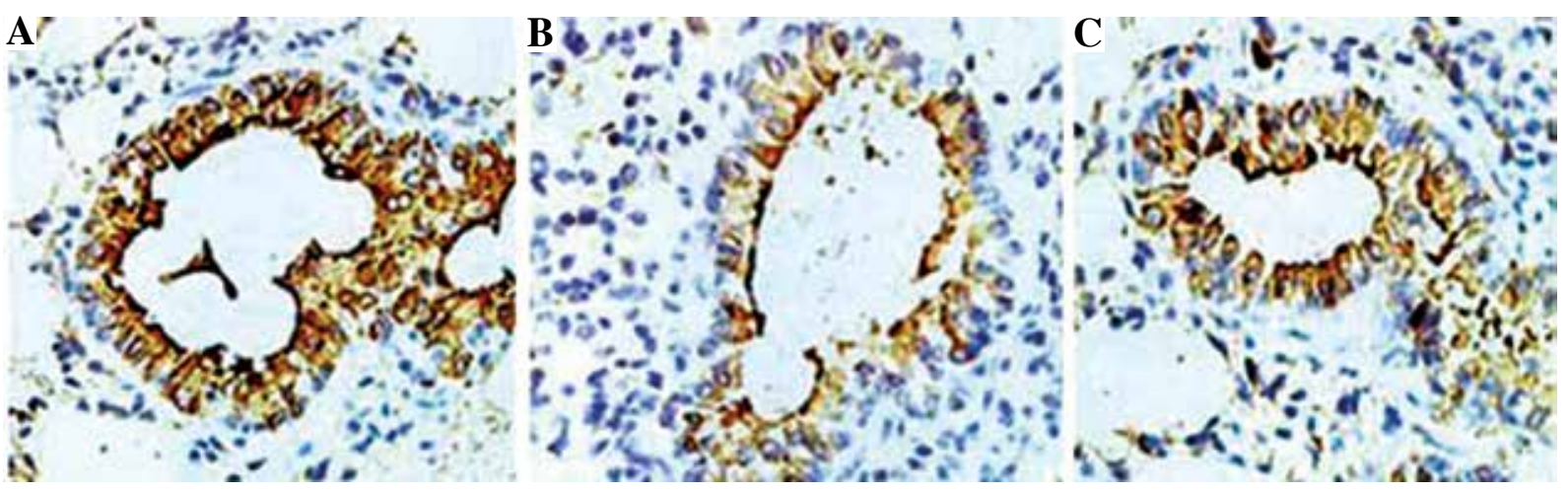

Fig. 3. Immunohistochemical staining for interleukin 12 ( $\times 400$ magnification). A) control group; B) model group; and C) dexamethasone group 

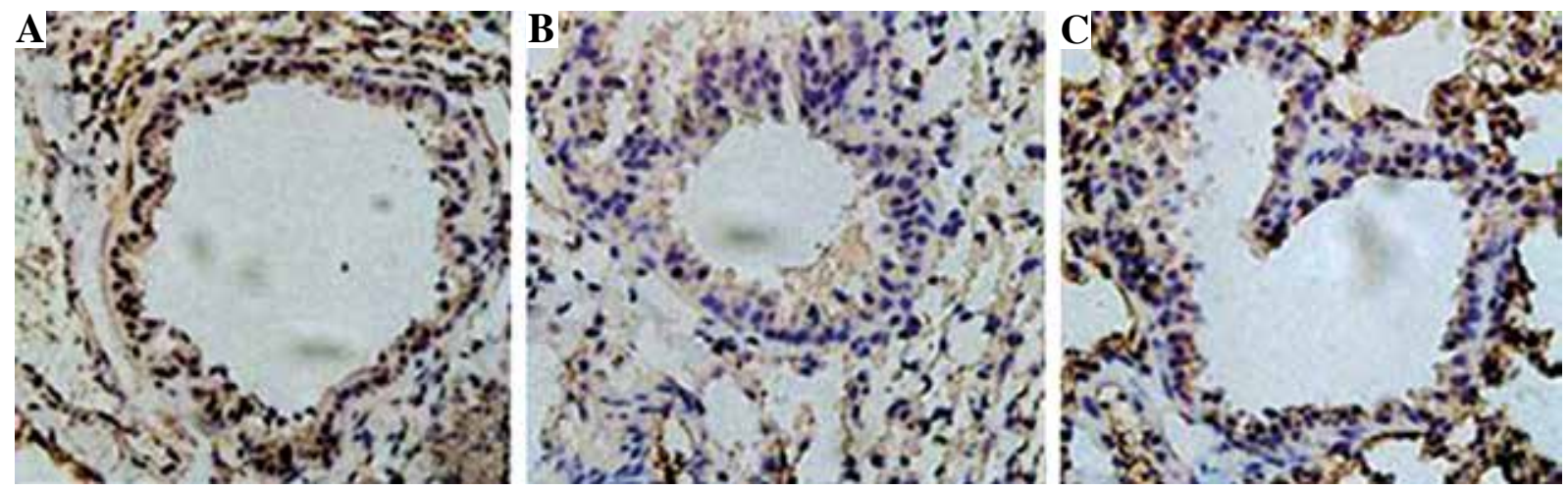

Fig. 4. Immunohistochemical staining for signal transducer and activator of transcription 4 ( $\times 400$ magnification). A) control group; B) model group; and C) dexamethasone group

NA155 [15, 16] can lead to changes in the airway, such as remodeling, thereby aggravating asthma symptoms.

In asthmatic mice, the alveolar wall is damaged by increased secretions. The number of inflammatory cells around the airway is significantly increased. However, injuries are improved after dexamethasone intervention. The total cell number, along with the number of EOS in BALF samples in asthmatic mice are significantly higher $(p<0.05)$ than those in normal mice; however, these numbers are significantly decreased $(p<0.05)$ following dexamethasone intervention, but still higher than those in normal mice. We found that miRNA-21 expression levels were significantly higher $(p<0.05)$ in asthmatic mice compared with those in normal mice. These miRNA levels were significantly decreased $(p<0.05)$ following glucocorticoid intervention, but still higher than those seen in control mice. Western blotting and immunohistochemistry results showed that IL-12 and STAT4 protein levels in the lungs of asthmatic mice were significantly reduced $(p<0.05)$ compared with those in control mice. Interleukin 12 and STAT4 protein levels were significantly improved $(p<0.05)$ after glucocorticoid intervention, but were still lower than those in control mice.

Recent studies have shown that miRNA-21 target sites are located within IL-12 mRNA, therefore miRNA-21 can significantly inhibit the translation process after IL-12 transcription [17]. Our findings indicate that miRNA-21 can regulate IL-12 and STAT4 translation post-transcriptionally, resulting in less IL-12 and STAT4 proteins. Our results reflect those of previous studies. Interleukin 12 is an important cell factor in maintaining the Th1/Th2 balance in the body [18-20]. The receptors are dimerized by combining with IL-12 receptors at the T0 cell surface, with JAK kinases activated by the dimerized receptor. This triggers phosphorylation of intracellular receptors that area tyrosine residues. Phosphorylated intracellular receptors begin to raise STAT4 levels and transfer it to the nucleus, where STAT4 is combined with the target gene promoter sequence to promote transcription of the target gene so that T0 cells are differentiated into Th1 cell subsets. The Th1 cell subsets can mediate the cellular immune response and the occurrence of delayed type hypersensitivity reactions. These results indicate that IL-12 can regulate the expression of STAT4, and that IL-12/ STAT4 signaling channels can inhibit asthma and other type I allergic reactions [21-24].

We have shown that miRNA-21 expression levels in asthmatic mice are significantly increased, and that miRNA-21 is specific to IL-12 mRNA allowing for inhibition of IL-12 translation. This miRNA also results in reduced STAT4 expression levels, which can lead to Th1/Th2 balance disorders. Type I allergic reactions can occur more easily under the stimulation of external allergens, thereby promoting the occurrence and development of asthma. The expression levels of miRNA-21 are decreased following dexamethasone intervention, such that IL-12 post-transcriptional translation is increased. When IL-12 expression levels are significantly increased, the expression level of STAT4 is increased, thereby promoting re-establishment of the Th1/ Th2 balance and reducing the incidence of asthma.

The authors declare no conflict of interest.

This study was supported by Grants of the Zhejiang Provincial Natural Science Foundation of China (No. LY12H10003), the Zhejiang Provincial Medical and Health Foundation of China (No. 2013KYB303), and the Lishui Nonprofit Technology Application Projects of Zhejiang Province of China (No. 2012JYZB29).

\section{References}

1. Vissing NH, Jensen SM, Bisgaard H (2012): Validity of information on atopic disease and other illness in young children reported by parents in a prospective birth cohort study. BMC Med Res Methodol 12: 160. 
2. Custovic A, Simpson A (2012): The role of inhalant allergens in allergic airways disease. J Investig Allergol Clin Immunol 22: 393-401; quiz follow 401.

3. Lommatzsch M (2012): Airway hyperresponsiveness: new insights into thepathogenesis. Semin Respir Crit Care Med 33: 579-587.

4. Pfitzner E1, Kliem S, Baus D, Litterst CM (2004): The role of STATs in inflammation and inflammatory diseases. Curr Pharm Des 10: 2839-2850.

5. Kim YS, Choi SJ, Choi JP, et al. (2010): IL-12-STAT4IFN- $\gamma$ axis is a key downstream pathway in the development of IL-13-mediated asthma phenotypes in a Th2 type asthma model. Exp Mol Med 42: 533-546.

6. Nathalie S, Jacinta B, Laure B, et al. (2013): IL-12 receptor $\beta 1$ deficiency alters in vivo $\mathrm{T}$ follicular helper cell response in humans. Blood 121: 3375-3385.

7. Munitz A, Brandt EB, Mingler M, et al. (2008): Distinct roles for IL-13 and IL-4 via IL-13 receptor alpha1 and the type II IL-4 receptor in asthma pathogenesis. Proc Natl Acad Sci U SA 105: 7240-7245.

8. Helene MN, Jorg T (2013): Epigenetic changes in inflammatory and autoimmune diseases. Springer Netherlands: Epigenetics: Development and Disease 61: 455-478.

9. Huang Y, Lei Y, Zhang H, et al. (2011): MicroRNA regulation of STAT4 protein expression: rapid and sensitive modulation of IL-12 signaling in human natural killer cells. Blood 118: 6793-6802.

10. Roh SS, Kim SH, Lee YC, Seo YB (2008): Effects of radix adenophorae and cyclosporine A on an OVA-induced murine model of asthma by suppressing to T cells activity, eosinophilia, and bronchial hyperresponsiveness. Mediators Inflamm 2008: 781425.

11. Seguin RM, Ferrari N (2009): Emerging oligonucleotide therapies for asthma and chronic obstructive pulmonary disease. Expert Opin Investig Drugs 18: 1505-1517.

12. Wong CF, Tellam RL (2008): MicroRNA-26a targets the histone methyltransferase Enhancer of Zeste homolog 2 during myogenesis. J Biol Chem 283: 9836-9843.

13. Chiba Y, Tanabe M, Goto K, et al. (2009): Down-regulation of miR-133a contributes to up-regulation of Rhoa in bronchial smooth muscle cells. Am J Respir Crit Care Med 180: 713719.

14. Hidalgo IJ, Borchardt RT (1990): Transport of bile acids in a human intestinal epithelial cell line, Caco-2. Biochim Biophys Acta 1035: 97-103.

15. O'Connell RM, Taganov KD, Boldin MP, et al. (2007): MicroRNA-155 is induced during the macrophage inflammatory response. Proc Natl Acad Sci U S A 104: 1604-1609.

16. Banerjee A, Schambach F, DeJong CS, et al. (2010): Micro-RNA-155 inhibits IFN-gamma signaling in CD4+ T cells. Eur J Immunol 40: 225-231.

17. Lu TX, Munitz A, Rothenberg ME (2009): MicroRNA-21 is up-regulated in allergic airway inflammation and regulates IL-12p35 expression. J Immunol 182: 4994-5002.

18. Freudenberg MA, Merlin T, Kalis C, et al. (2002): Cutting edge: a murine, IL-12-independent pathway of IFN- $\gamma$ induction by Gram-negative bacteria based on STAT4 activation by type I IFN and IL-18 signaling. J Immunol 169: 1665-1668.

19. Zielonka MT, Demkow U, Filewska M, et al. (2007): Angiogenic activity of sera from interstitial lung diseases patients in relation to IL-6, IL-8, IL-12 and TNF $\alpha$ serum level. Centr Eur J Immunol 32: 53-60.
20. Rossi A, Cantisani C, Carlesimo M, et al. (2012): Serum concentrations of IL-2, IL-6, IL-12 and TNF-alpha in patients with alopecia areata. Int J Immunopathol Pharmacol 25: 781-788.

21. Afanasyeva M, Wang Y, Kaya Z, et al. (2001): Interleukin-12 receptor/STAT4 signaling is required for the development of autoimmune myocarditis in mice by an interferon-gamma-independent pathway. Circulation 104: 3145-3151.

22. Lund RJ, Chen Z, Scheinin J, Lahesmaa R (2004): Early target genes of IL-12 and STAT4 signaling in Th cells. J Immunol 172: 6775-6782.

23. Sondergaard JN, Brix S (2012): Isolation of IL-12p70-competent human monocyte-derived dendritic cells. J Immunol Methods 386: 112-116.

24. Cheng X, Folco EJ, Shimizu K, Libby P (2012): Adiponectin induces pro-inflammatory programs in human macrophages and CD4+ T cells. J Biol Chem 287: 36896-36904. 